\title{
Diffuse Meningiomatosis without Neurofibromatosis: A Rare Diagnosis with Atypical Presentation
}

\author{
Smily Sharma ${ }^{1}$ Pankaj Sharma $^{1}$ Amit Kumar ${ }^{1}$ \\ ${ }^{1}$ Department of Radiodiagnosis and Imaging, All India Institute of \\ Medical Sciences, Rishikesh, Uttarakhand, India \\ Indian J Radiol Imaging 2021;31:768-771.
}

Address for correspondence Pankaj Sharma, Department of Radiodiagnosis and Imaging, All India Institute of Medical Sciences, Rishikesh, Uttarakhand, India (e-mail: pankajrad7477@yahoo.com).

\author{
Abstract \\ Keywords \\ - en plaque \\ meningioma \\ - meningiomatosis \\ - neurofibromatosis 2
}

Meningiomas are amongst the most common neoplasms of the central nervous system; however, "multiple meningiomas" or "meningiomatosis" account for $<10 \%$ of cases. The association with neurofibromatosis 2 is seen in $\sim 50 \%$ of cases. We report a case of 35-year-old female patient who presented with left eye proptosis, left forehead swelling, and multiple cranial nerve palsies predominantly on the left side. Imaging evaluation revealed innumerable en plaque meningiomas forming a sheet-like nodular thickening along the dura, causing marked hyperostosis and extending into the orbits, cavernous sinuses, sellar-suprasellar regions, various skull foramina, basal cisterns and into the cervical spinal canal causing mass effect on vital structures of the brain and cervical spinal cord. Similar lesions were found scattered in rest of the spine. We intend to highlight the role of imaging in accurately establishing the diagnosis and evaluating the extent and burden of disease in such rare cases.

\section{Introduction}

Meningiomatosis refers to the presence of more than two meningiomas occurring at the same time at two different locations. ${ }^{1}$ Multiple meningiomas can be discrete or can rarely occur as diffuse involvement of meninges. ${ }^{2}$ Its association with neurofibromatosis 2 (NF 2) has been established but sporadic occurrence has also been reported. Although meningioma is the most common intracranial benign tumor, meningiomatosis accounts for only 1 to $10 \%$ of cases. ${ }^{3}$ Given the rarity of diagnosis, we report a case of mid-aged female with no family history or stigmata of NF 2 who presented with left eye proptosis and hearing loss and was found to have innumerable en plaque supratentorial, infratentorial, and spinal meningiomas. Other clinical and imaging findings required for the diagnosis of neurofibromatosis were not found in the case.

\section{Case Description}

A 35-year-old female patient presented to our institution with bilateral hearing loss since 8 months. She had undergone a neurosurgical intervention at 18 years of age but no records were presently available. No relevant family history could be ascertained. On examination, she had proptosis of the left eye and swelling over the left side of the forehead (-Fig. 1). No cutaneous stigmata of neurofibromatosis were present. She had features of multiple cranial nerve palsies predominantly involving the left side. On pure tone audiometry, she was found to have bilateral sensorineural hearing loss (left $>$ right). She underwent contrast-enhanced magnetic resonance imaging (MRI) brain and screening of the whole spine (Siemens Magnetom Aera; 1.5T MRI Scanner) and non-contrast computed tomography (CT) scan (Philips Ingenuity Core 64 Slice CT Scanner) in our department.
DOI https://doi.org/ 10.1055/s-0041-1736394. ISSN 0971-3026. (c) 2021. Indian Radiological Association. All rights reserved.

This is an open access article published by Thieme under the terms of the Creative Commons Attribution-NonDerivative-NonCommercial-License, permitting copying and reproduction so long as the original work is given appropriate credit. Contents may not be used for commercial purposes, or adapted, remixed, transformed or built upon. (https://creativecommons.org/ licenses/by-nc-nd/4.0/)

Thieme Medical and Scientific Publishers Pvt. Ltd., A-12, 2nd Floor, Sector 2, Noida-201301 UP, India 


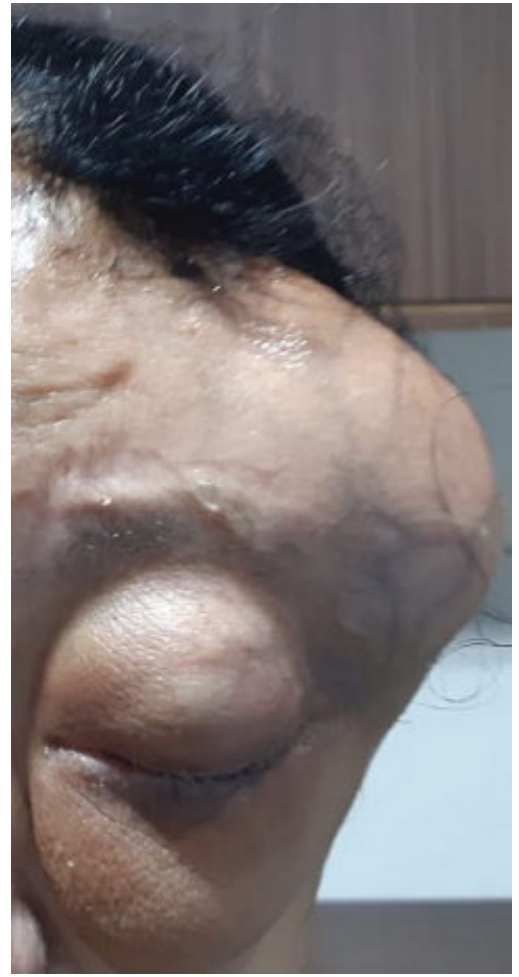

Fig. 1 Clinical picture of a 35-year-old female patient who presented with complaints of left eye proptosis and left forehead swelling.

Innumerable well-defined extra-axial dural-based round to lobulated mass lesions were seen along the dura and falx cerebri in the midline as well as lining the cerebral hemispheres bilaterally. The lesions were iso to hyperintense on T1WI/T2WI/FLAIR and showed marked post-contrast enhancement. A few of the lesions showed mild diffusion restriction and blooming on susceptibility-weighted imaging (SWI).

Similar signal characteristic lesions were seen contiguously in the form of extensive sheet-like nodular thickening along the dura, predominantly on the left side in the sellar, suprasellar, interpeduncular, mesencephalic, left CP angle cistern, left jugular foramen, left hypoglossal canal, along the clivus, and extending through the foramen magnum into cervicomedullary and upper cervical region. The lesions were compressing the pituitary, brain stem, left cerebellar hemisphere, involved cranial nerves, cervicomedullary junction, and cervical spinal cord.

The lesions in the sellar and suprasellar regions were infiltrating into bilateral cavernous sinuses and extending into the left perisylvian region, where the M1 segment of left middle cerebral artery was encased. The lesions extended through the left superior orbital fissure and the left optic canal into the left orbit causing encasement, compression, and thinning of the left optic nerve. Proptosis of the left eye was seen with infiltration of the subcutaneous tissue of the left inferior eyelid and infraorbital region (-Figs. 2-4).

Non-contrast CT scan of the brain showed the presence of the extra-axial lesions with expansion and marked hyperostosis involving bony calvarium, base of the skull on the left side, sella, clivus, left mastoid, left orbital walls, and leftsided zygomatic arch (- Fig. 5).

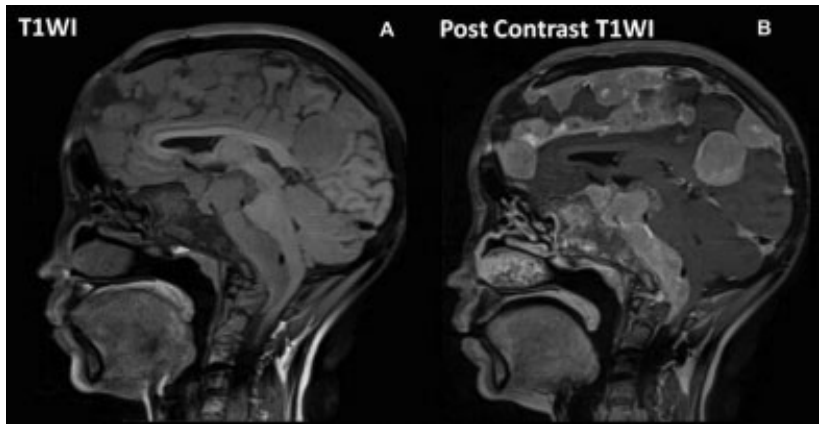

Fig. 2 (A and B) Sagittal T1WI and post-contrast T1WI MRI images showing innumerable extra-axial dural-based masses along the falx cerebi, sellar-suprasellar region, basal cisterns, and extending through the foramen magnum into THE upper cervical region. The lesions are causing compression of the pituitary, brain stem, and cervical spinal cord (arrow).

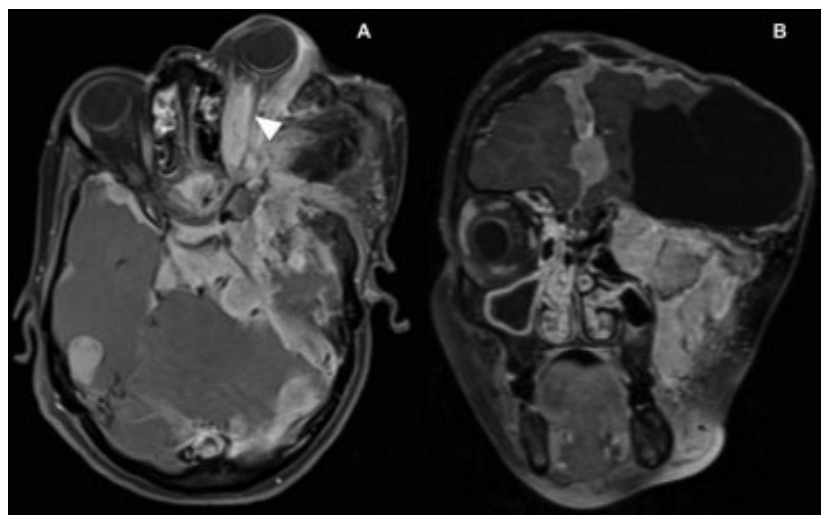

Fig. 3 (A) Axial postcontrast T1WI showing the lesions in the sellar and suprasellar regions extending into the left perisylvian region and into the left orbit through the superior orbital fissure and the optic canal and causing compression of the left optic nerve (arrow head). (B) Coronal postcontrast T1WI showing the lesions in the left orbit infiltrating into the left periorbital region. Also, note the nodular thickening along the falx cerebri.

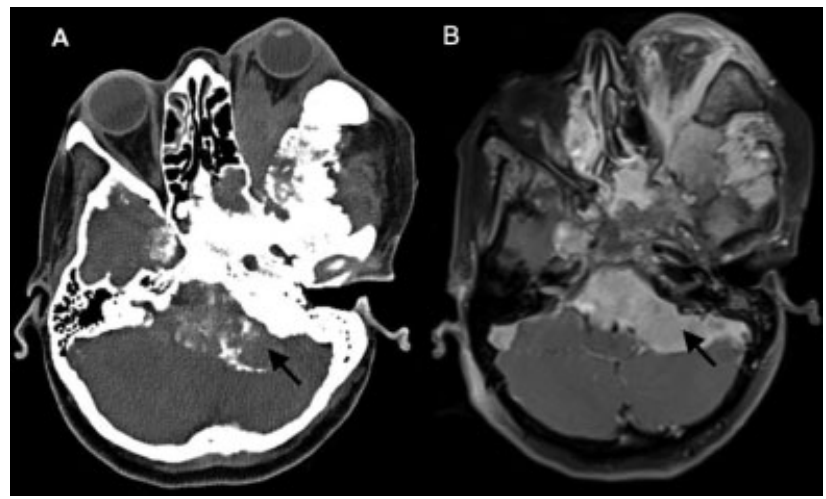

Fig. 4 (A) Axial non-contrast CT image of the brain showing calcified dural-based lesions along the prepontine and cerebellopontine (CP) angle cisterns (arrow). (B) Axial postcontrast T1WI MRI image showing intensely enhancing dural-based lesions involving the left $C P$ angle and prepontine cisterns and extending up to the right cerebellopontine cistern (arrow). 


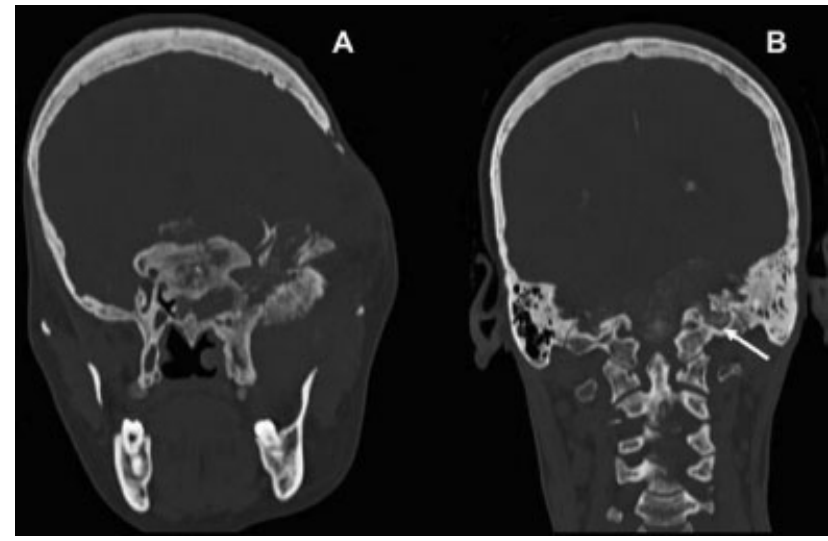

Fig. 5 Marked hyperostosis along the skull seen on NCCT further confirmed the diagnosis of en plaque meningiomatosis. Calcification is seen in a few lesions. A few lesions were extending into the left jugular foramen (arrow) and left internal acoustic meatus (not shown).

Left frontal and left superior orbital walls were deficient due to previous craniotomy, and a cystic lesion was seen herniating through the defect. The cystic lesion had thin nonenhancing septations, and it was continuous with the frontal horn of left lateral ventricle suggestive of porencephalic cyst formation. Ultrasound forehead (GE Logic S8; Curvilinear Array: 3-5 MHZ) showed a cystic lesion with thin internal septations and underlying herniation of the brain parenchyma (-Fig. 6).

Screening of the spine showed multiple enhancing intradural extramedullary dural based lesions scattered throughout the spine, with the lesions at C5-C6 and D11-D12 levels causing spinal canal stenosis. No extension into or widening of any neural foramina was seen, ruling out the possibility of neurofibroma (-Fig. 7).

Summarizing our case, imaging revealed the presence of innumerable dural-based enhancing lesions in the brain and spinal cord, which led to the compression of vital intracranial

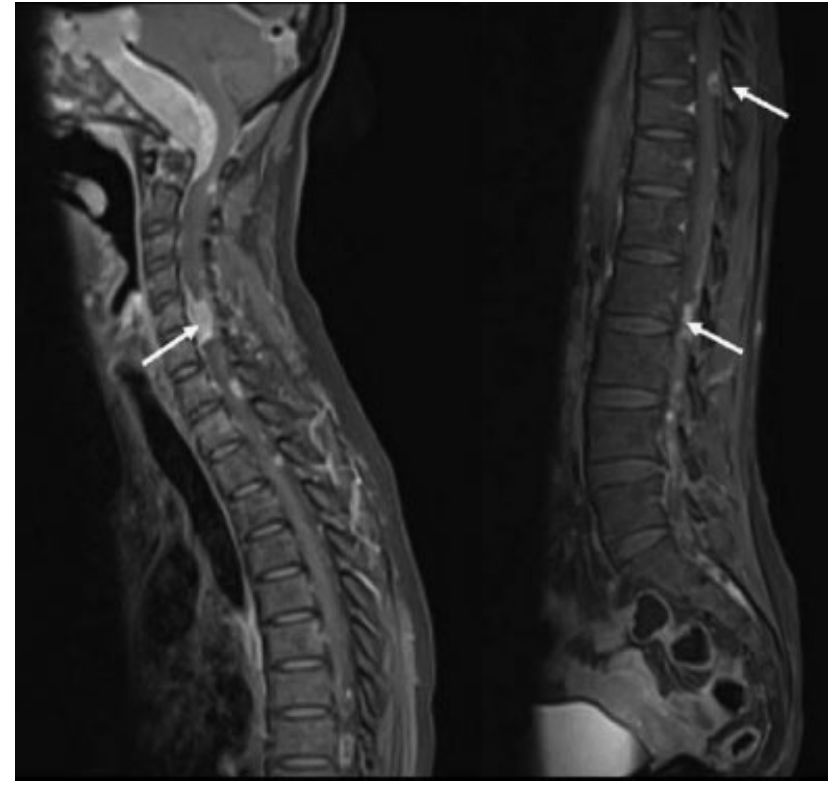

Fig. 7 Post-contrast T1WI MRI images of the whole spine showing intradural extramedullary dural-based enhancing lesions with associated dural tail (seen in the largest lesion) and no widening or extension into the neural foramina, suggestive of meningiomas (arrows).

and intra-orbital structures and spinal canal stenosis. The extra-axial location of the lesions, sheet-like nodular thickening of the dura, associated marked calvarial hyperostosis with no bony erosions, presence of calcification, and intense post-contrast enhancement were suggestive of features of "diffuse meningiomatosis with multiple en plaque meningiomas in the brain., ${ }^{4,5}$ The intradural extramedullary location without any extension into the neural foramina or any widening of the foramina helped to clinch the diagnosis of spinal meningiomas. The mass effect on cranial nerves and extension into the left internal acoustic meatus with associated hyperostosis explained the presence of multiple cranial

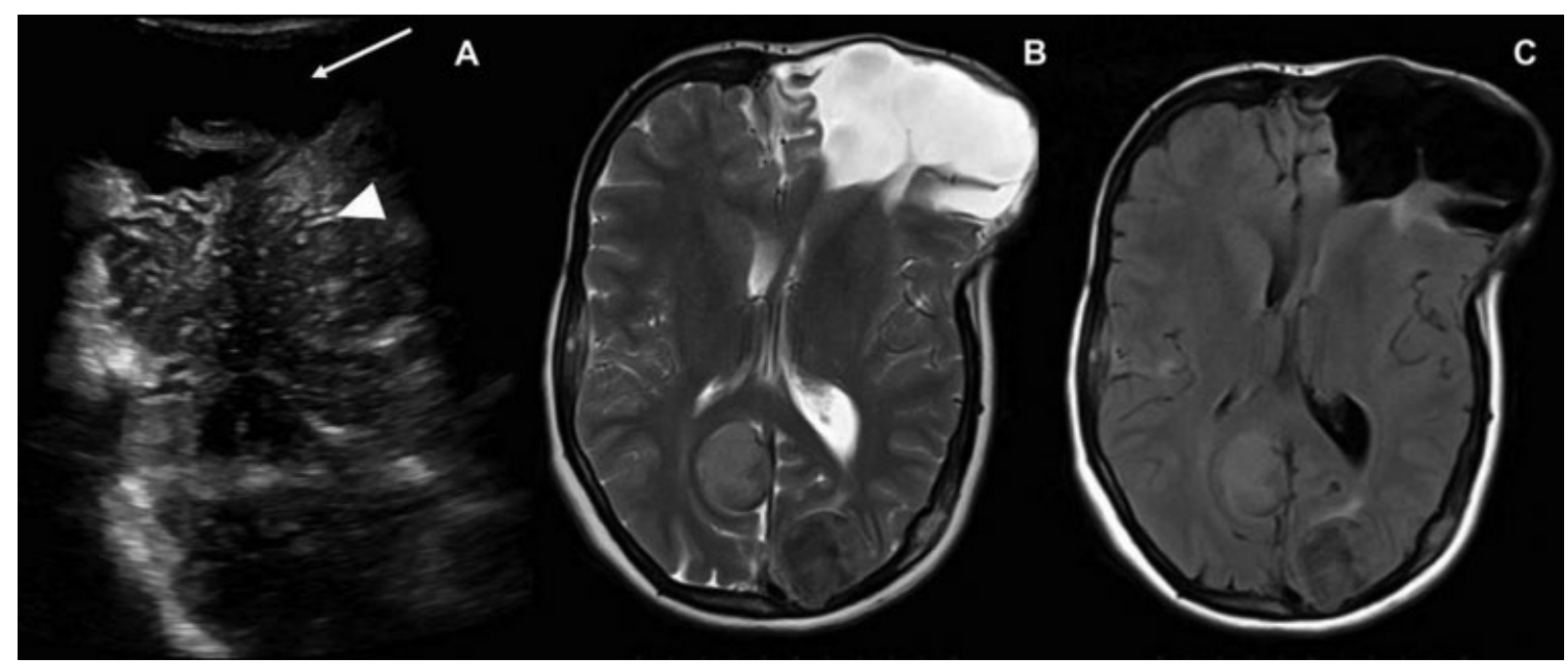

Fig. 6 (A) Ultrasound image (curvilinear array) through the forehead swelling showing a cystic lesion (arrow) with underlying brain parenchyma (arrowhead). (B and C) Axial T2WI and corresponding FLAIR MRI images showing a cystic lesion with the surrounding FLAIR hyperintense area in the left frontal region. The lesion is communicating with the frontal horn of the left lateral ventricle, suggestive of a porencephalic cyst formation. 
nerve palsies with left-sided hearing loss. No other neoplasms such as schwannoma or ependymoma were evident to suggest the classic "MeSMe" association of NF 2.

Due to the involvement of vital brain structures and thereby the risk involved, the patient decided not to undergo surgery. She was referred for radiotherapy and kept on regular followup. However, she did not show significant improvement.

\section{Discussion}

Coined by Cushing and Eisenhardt, "multiple meningiomas" or meningiomatosis refers to "the presence of two or more spatially separated meningiomas without the features of neurofibromatosis." ${ }^{1}$ Although meningioma is the most common intracranial benign tumor, meningiomatosis accounts for only $<10 \%$ of the cases. ${ }^{6}$

The pathophysiology has been poorly understood. However, they are associated with NF 2 in $\sim 50 \%$ of cases. The other cases include sporadic and familial varieties and those that occur as a consequence of spread from a solitary meningioma. A hypothesis describing their occurrence states that they arise as independent tumors. Another hypothesis states that they arise due to a single clonal proliferation and consequently spread via the cerebrospinal fluid. ${ }^{6-8}$ Association with other syndromes such as Cowden syndrome, Werner syndrome, and germline SMARCB1 mutation have also been reported. The SMARCB1 mutation predisposes to both schwannomatosis and meningiomatosis. ${ }^{9}$

Meningiomatosis has a stronger female predilection (3.5:1) than solitary meningiomas (2:1). Occurrence in children and adolescents is less common. They may be incidentally detected or can present as space-occupying lesions. The most common location is along the convexities, with hemicranial involvement being more common. Infratentorial location and involvement of the posterior fossa are very rare. ${ }^{6,7,10}$ Our case was also a female patient with predominant left side involvement but was an exception in the regard that innumerable ( $>50$ ) meningiomas were present in both supra and infratentorial locations and in the spinal canal.

Our case was also unique due to the presence of innumerable sheet such as en plaque meningiomas involving various vital structures of the brain and showing extension to the orbit and infiltration of periorbital soft tissue. Only a few such cases of en plaque meningiomas with diffuse hyperostosis and extension to subcutaneous tissue have been reported in the literature. ${ }^{4,5,11}$

Histopathologically, the majority of solitary and multiple meningiomas are WHO Grade 1 with meningothelial subtype ${ }^{6,8,12}$ Although multiple, no significant increase in malignant potential or growth rate is seen with multiple meningiomas.

The management options include surgical resection, radiation therapy, and stereotactic radiosurgery, with surgery being the treatment of choice. Its indications include symptomatic cases measuring $>3 \mathrm{~cm}$ in size, faster growth rate, and peritumoral edema with surgical accessibility. ${ }^{1,6}$ Overall prognosis with multiple meningiomas is good with low rates of morbidity and mortality. In a review by Pereira et al, a recurrence rate of $\sim 8 \%$ was found after treatment. ${ }^{6}$ Recommendations about follow-up have not been well specified although long-term surveillance may be required due to possible late-onset recurrence. ${ }^{13}$

\section{Conclusion}

Although solitary meningiomas are very common, the occurrence of innumerable $(>50)$ meningiomas in both supra as well as infratentorial locations and in the spinal canal in the form of diffuse meningeal involvement without any evidence of NF 2 is very rare. Imaging plays a pivotal role in establishing the diagnosis and describing the extent and mass effect on vital cranial structures and spinal cord caused by diffuse extensive meningiomatosis.

Funding

None.

Conflicts of Interest

None declared.

\section{References}

1 Koech F, Orege J, Ndiangui F, Macharia B, Mbaruku N. Multiple intracranial meningiomas: a review of the literature and a case report. Case Rep Surg 2013;2013:131962

2 Harbitz H. A case of multiple meningiomas combined with diffuse meningiomatosis. Acta Pathol Microbiol Scand 2010;12:24-37

3 Ohla V, Scheiwe C. Meningiomatosis restricted to the left cerebral hemisphere with acute clinical deterioration: case presentation and discussion of treatment options. Surg Neurol Int 2015;6:64

4 Akutsu H, Sugita K, Sonobe M, Matsumura A. Parasagittal meningioma en plaque with extracranial extension presenting diffuse massive hyperostosis of the skull. Surg Neurol 2004;61(02): 165-169, discussion 169

5 Kim KS, Rogers LF, Goldblatt D. CT features of hyperostosing meningioma en plaque. AJR Am J Roentgenol 1987;149(05):1017-1023

6 Araújo Pereira BJ, Nogueira de Almeida A, Pires de Aguiar PH, Paiva WS, Teixeira MJ, Nagahashi Marie SK. Multiple intracranial meningiomas: a case series and review of the literature. World Neurosurg 2019;122:e1536-e1541

7 Chaurasia B, Hossain M, Barua K, et al. Meningiomatosis in nonneurofibromatosis patient: how we dealt with 8 tumors. J Neurol Stroke 2018;8:307-310

8 Koh YC, Yoo H, Whang GC, Kwon OK, Park HI. Multiple meningiomas of different pathological features: case report. J Clin Neurosci 2001;8(Suppl 1):40-43

9 Christiaans I, Kenter SB, Brink HC, et al. Germline SMARCB1 mutation and somatic NF2 mutations in familial multiple meningiomas. J Med Genet 2011;48(02):93-97

10 Nagano T, Saiki I, Kanaya H. Multiple meningiomas in the posterior fossa. Surg Neurol 1985;23(04):425-427

11 Hashimoto H, Hirabayashi H, Hiramatsu K, MorimotoT, Tsunoda S, Sakaki T. Malignant parasagittal meningioma appearing as a large subcutaneous mass [article in Japanese]. No Shinkei Geka 1994; 22(08):785-788

12 Neuss M, Westphal M, Hänsel M, Herrmann HD. Clinical and laboratory findings in patients with multiple meningiomas. $\mathrm{Br} \mathrm{J}$ Neurosurg 1988;2(02):249-256

13 Nakamura M, Roser F, Michel J, Jacobs C, Samii M. The natural history of incidental meningiomas. Neurosurgery 2003;53(01): 62-70, discussion 70-71 